\title{
The Functional and Experimental Model for Extenics Simulations of the Mobile Robots
}

\author{
Daniel Mitroi, Luige Vladareanu, Victor Vladareanu, Hongbo Wang, Yongfei Feng, and Jianye Niu
}

\begin{abstract}
The paper presents the functional and experimental model for Extenics simulations of the mobile robots applied on VIPRO Platform. A brief history and overview of the relevant theoretical concepts is provided. Intelligent interface for extended control use a new component for artificial intelligence which is extended control (Extenics) to improve DHFPC control instantaneous performance of mobile robots. Applying Extenics own techniques and implementation in a fuzzy space results a new, innovative application for the VIPRO platform which can be used to simulate and implement new control methods of mobile robots.
\end{abstract}

Index Terms-Extenics control, intelligent control interfaces, tracking trajectory control, contradictory problems.

\section{INTRODUCTION}

Extenics is a novel theoretical frame of matter element in the world leading science, which establishes the methodology of solving contradictory problems through the intelligent and innovative process in a formal, mathematical and logical way.

The quantitative tools to solve the robotics contradictory problems based on extension set of extension mathematics are examined. Extension decision methods on robot control field are done by applying a series of comprehensive strategies using extension set and dependent function in order to transformations of goal matter-elements or condition matter-elements in consistency elements and rules of cognitive reasoning. The robotics intelligent control by extension theory and the basic concepts, structure and principles of extension control developed as the knowledge base, the characteristics pattern recognition, fusion of dependent degree on characteristic states, the measure pattern recognition, the adaptive mechanism, the control strategies as the main elements of the control system architecture.

Extenics was developed by Cai Wen in 1983 and developed successively, with a major impact in the scientific world of the last few years through results in e-learning, data mining, image recognition, robotics, statistics and management research, among others [1], [2], [8], [11]. Extension set theory is a mathematical form for representing uncertainty which is an extension of classic set theory, with applications in many research fields [13]-[16]. Extenics is a

Manuscript received April 21, 2017; revised June 15, 2017.

Daniel Mitroi, Luige Vlădăreanu and Victor Vlădăreanu are with Romanian Academy, Institute of Solid Mechanics, RO (e-mail: vladareanuv@gmail.com).

Hongbo Wang, Yongfei Feng, and Jianye Niu are with Yanshan University, Parallel Robot and Mechatronic System Laboratory of Hebei Province, Qinhuangdao, 066004, China (e-mail: hongbo_w@ysu.edu.cn). field of study which aims to solve contradictory problems, as is the case of position - force control in the field of robotics, mechatronics and real time control.

\section{VIPRO PLATFORM ARCHITECTURE}

The technical solution for the new platform presented in Fig. 1 includes modules of 3D VIPRO platform designed work in an open architecture with real time control [12]. Intelligent control interfaces use advanced control strategies based on artificial intelligence algorithms adapted to the type of robot and to the operating environment. The used types of control are: extended control (extenics), neutrosophic control, human adaptive control and these control types are implemented using IT\&C real time, rapid processing communications. On the VIPRO platform, the following interfaces were designed: extended control interface, robot neutrosophic control interface and neural nets interface for force-position hybrid dynamic control [1], [3], [4], [6], [12].

VIPRO platform is a complex device which allows development of new control, flexible mobile interfaces in a simulated environment that can be validated on a physical system. Physical systems are actually real time controlled robots or mechatronic systems capable of replacing human operators in highly risk zones. Control interfaces have been the subject for researches in robotics and the results never ceased to appear. Part of these results were the mechatronics systems with detection, transport and manipulation capabilities for a variety of application.

In this context, it is necessary to simulate mechatronic systems and to choose their proper algorithms as well. Mobile robot's 3D simulation has to reproduce the real system dynamics and the environment, as accurate as possible. Another aspect to be taken into account when the 3D model is created is the interaction between various mechatronic systems that can change the dynamic for the new resulted system [9], [10].

\section{EXTENICS INTELLLIGENT CONTROL INTERFACES}

Extenics, the real-time control method is integrated in this type of interface. The theory behind this method is the theory of the same name, Extenics, theory that helps to solve contradictory problems. It is why it is applied in extended system control by applying metrics and dependencies function, defined by extenics logic. The initiator or this method is Prof. Cai Wen [1], [2] from Guangdong University in 1983. Subsequent iteration, with significant contributions has been made by Prof. Smarandache, the University of New 
Mexico, USA and Prof. Şandru, the Polytechnic University of Bucharest, both members of the research team of the project VIPRO. Improvements consist in transition from an 1-dimension laniary space to an $\mathrm{n}$-dimension laniary space [6], [7].

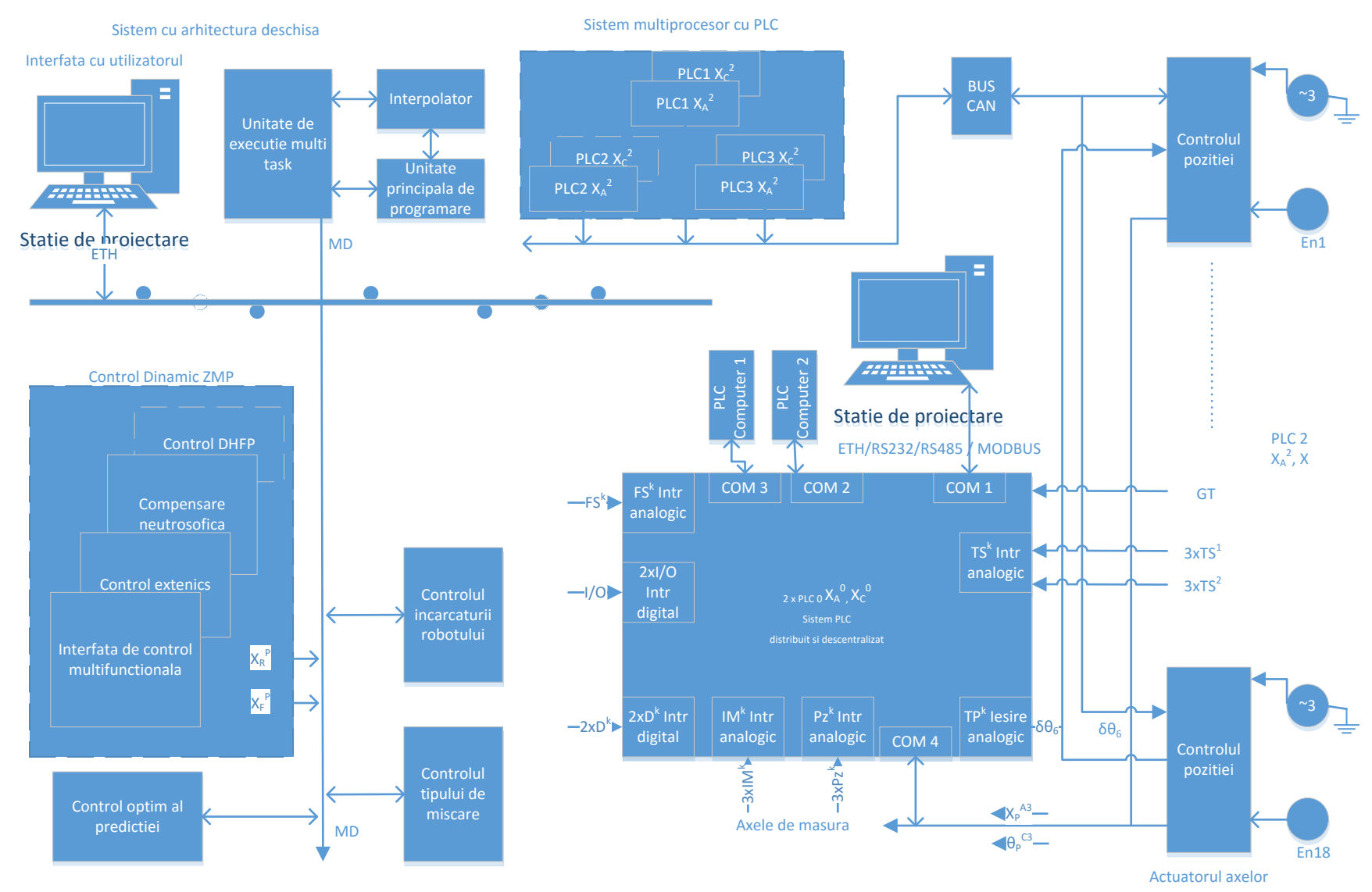

Fig. 1. 3D VIPRO platform - Open architecture system using intelligent interfaces.

It was solved contradictory force-position control of robot motion in real time using VIPRO platform. It has thus been extended control of the hybrid type used force-position eHFPC, the method developed by Prof. Vlădăreanu [3], [6[-[8]. Real-time operation involves control interface implementation of the patented method [11] in the VIPRO platform.

The intelligent interface for extended control (ICEx) develops a new component of intelligent interface, the extended control (Extenics) order to improve DHFPC control instantaneous performance of mobile robots. Applying contradictory problems solving techniques (Extenics) in a fuzzy space is leading to a new innovative application for the VIPRO platform, presented below, which allows modeling and implementation of a new control method of mobile robots.

Modeling control techniques based on Extenics and $\mathrm{nD}$ space norms. It is assumed that we have a robotic application determined by a work space of a mechatronic mechanism which contains a punctual, bi-dimensional reference - on ( $\mathrm{x}$, y) axes - defined as target for the robotic end-effector.

For the actuators control we assume the usage of an extended control which implements the concepts presented in Extenics theory. To achieve it, it is necessary to know the dependency function, calculated in a multidimensional space to estimate the incompatibility level that will be used in choosing the actuator intensity response. This procedure will be explained in detail in the sections dedicated to extended control. To simulate robotic space, it is necessary to calculate the dependency function for multidimensional cases.

Using theories developed in parallel by professors Smarandache and Şandru, regarding n-dimensional spaces function, we obtain a unitary expression of the dependency function for the studied case. Starting with the reference point $\mathrm{R}(\mathrm{xr}, \mathrm{yr}$ ) which is the optimum point for the controlled position of the actuator, we have an accepted reference interval X0 and an acceptable interval $\mathrm{X}$ and for both of them the reference is R [8]-[11].

To find extended indicators expression we will consider two regions of a bi-dimensional space. The two zones correspond to space variations on $\mathrm{x}$ and $\mathrm{y}$ axes. For any point $\mathrm{P}(\mathrm{x} \neg \mathrm{P}, \mathrm{yP})$ it can be calculated (according Prof. Smarandache) the extended 2D distances related to existent intervals:

$$
\begin{gathered}
\rho(x, X)=\left|P P_{2}\right| \\
\rho\left(x, X_{0}\right)=\left|P P_{1}\right|
\end{gathered}
$$

The resulting dependency function is shown below:

$$
k(P)=\frac{\rho(x, X)}{D\left(x, X_{0}, X\right)}=\frac{\rho(x, X)}{\rho(x, X)-\rho\left(x, X_{0}\right)}=\frac{\left|P P_{2}\right|}{\left|P P_{2}\right|-\left|P P_{1}\right|}
$$


where $\mathrm{P}$ is chosen to be outside of $\mathrm{X} 0$, but included in $\mathrm{X}$ and the result is a negative dependence subunit function (function denominator is negative). If it belonged to the $\mathrm{X} 0, \mathrm{k}(\mathrm{Q})$ would be calculated in the same way, but the result would be positive, and if $\mathrm{P}$ would be outside of $\mathrm{X}$, the dependency function would be smaller than -1 .

The value of the dependency function in the point $\mathrm{P}$ will be the extended distance in 2D space between the point and the nearest border, divided by the difference between the point and the big interval, in one side, and the distance between the point and the small interval, on the other side. All these distances are taken on the line between the optimal point $\mathrm{R}(\mathrm{x}, \mathrm{y})$ and the chosen point $\mathrm{P}(\mathrm{xP}, \mathrm{yP})$.

According to Vlădăreanu-Smarandache method [9] (F. Smarandache, L. Vlădăreanu, 2012) it will determine classes on points on vertically with the same value for the dependency function in this space, because the final expression will not depend on the y coordinate value in the selected point. For any point $\mathrm{Q}(\mathrm{xQ}, \mathrm{yQ})$ will result a final expression, similar to the previous one, [8], where

$$
k(Q)=\frac{\rho(x, X)}{D\left(x, X_{0}, X\right)}=\frac{\rho(x, X)}{\rho(x, X)-\rho\left(x, X_{0}\right)}=\frac{\left|Q Q_{2}\right|}{\left|Q Q_{2}\right|-\left|Q Q_{1}\right|}
$$

This zone will determine classes of horizontal points with the same value of dependency function. Thus, it can be observed that, for such a repartition of the extended interval which are the basis of the dependency function, the bi-dimensional problem can be split in two one-dimensional distinctive problems.

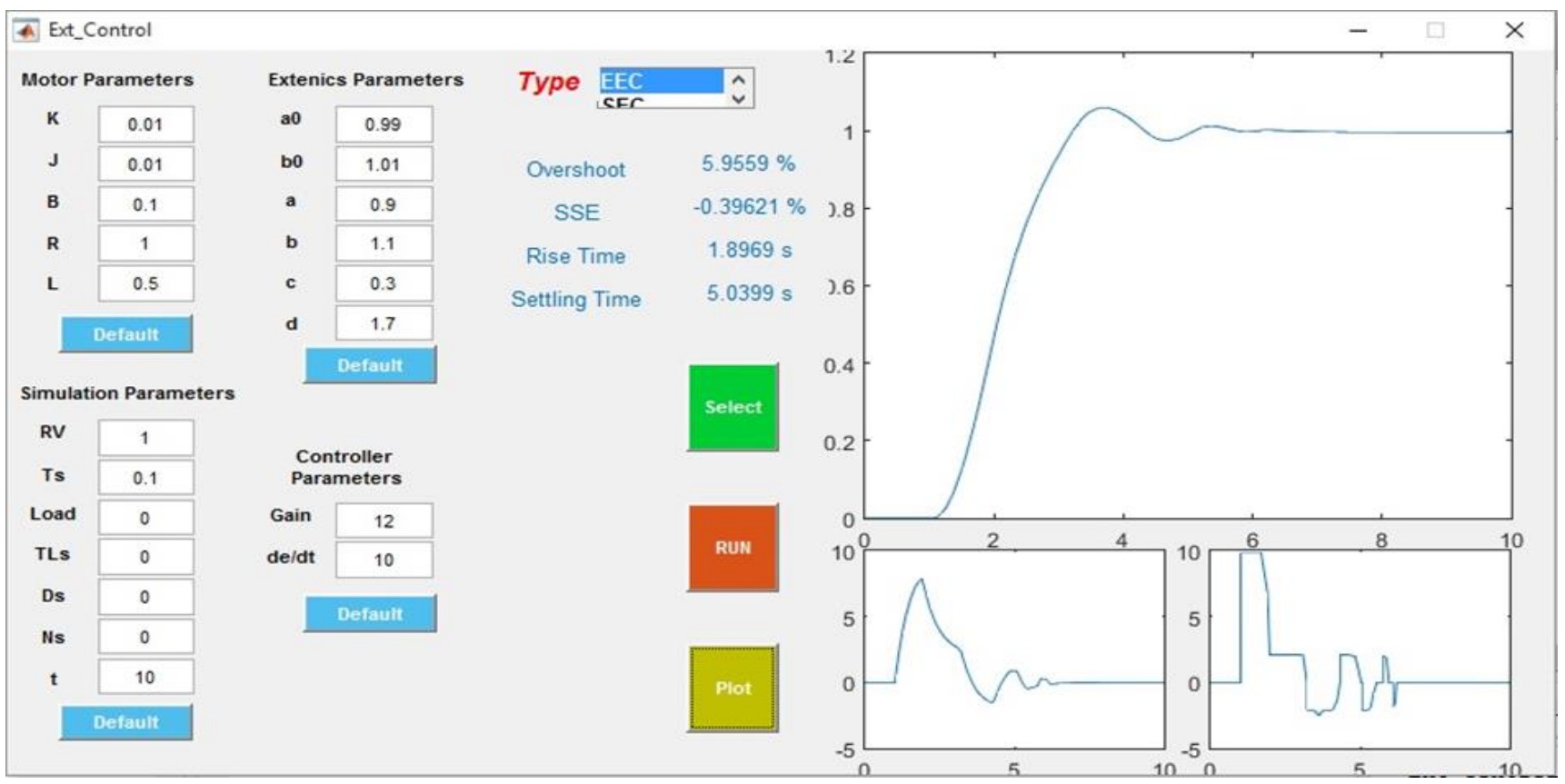

Fig. 2. Extended control interface.

To develop robotic control, including rescue ones, using the VIPRO virtual robot platform, it has been developed the experimental and functional model for intelligent interface of extended control (ICEx) as a visual application that can be called from local or remote VIPRO platform. The application allows simulation, testing and optimization of extended control strategies for VIPRO project. The application is developed in Matlab programming language, using visual development tools to design a new user interface taking into consideration fuzzy/extenics modelling tools from Matlab. In the Fig. 2, graphical elements and control buttons are presented.

For each category of parameters, there is an option to upload a set of standard values for rapid testing of the interface or activities to correct any code errors (bugs).

Parameters used to run the simulation, which will be loaded in Simulink, are:

- RV: the reference value (a scaling factor for control space)
- Ts: the continuous process discretization (time sample)

- Load: the engine load (it can be either positive or negative)

- TLs: the time when the load is applied

- Ds: the system disturbance applied on the control value

- Ns: the system noise applied on the reaction loop

- $\mathrm{t}$ : simulation time

Characteristic parameters for an extended regulator can be specified, i.e. the intervals defining extended control zones:

$$
(c, d) \supset(a, b) \supset\left(a \_0, b \_0\right)
$$

where:

- a0: left limit of the compatibility zone

- b0: right limit of the compatibility zone

- a: left limit of the transforming zone

- b: right limit of the transforming zone

- c: left limit of the incompatibility zone

- $\quad$ d: right limit of the incompatibility zone 


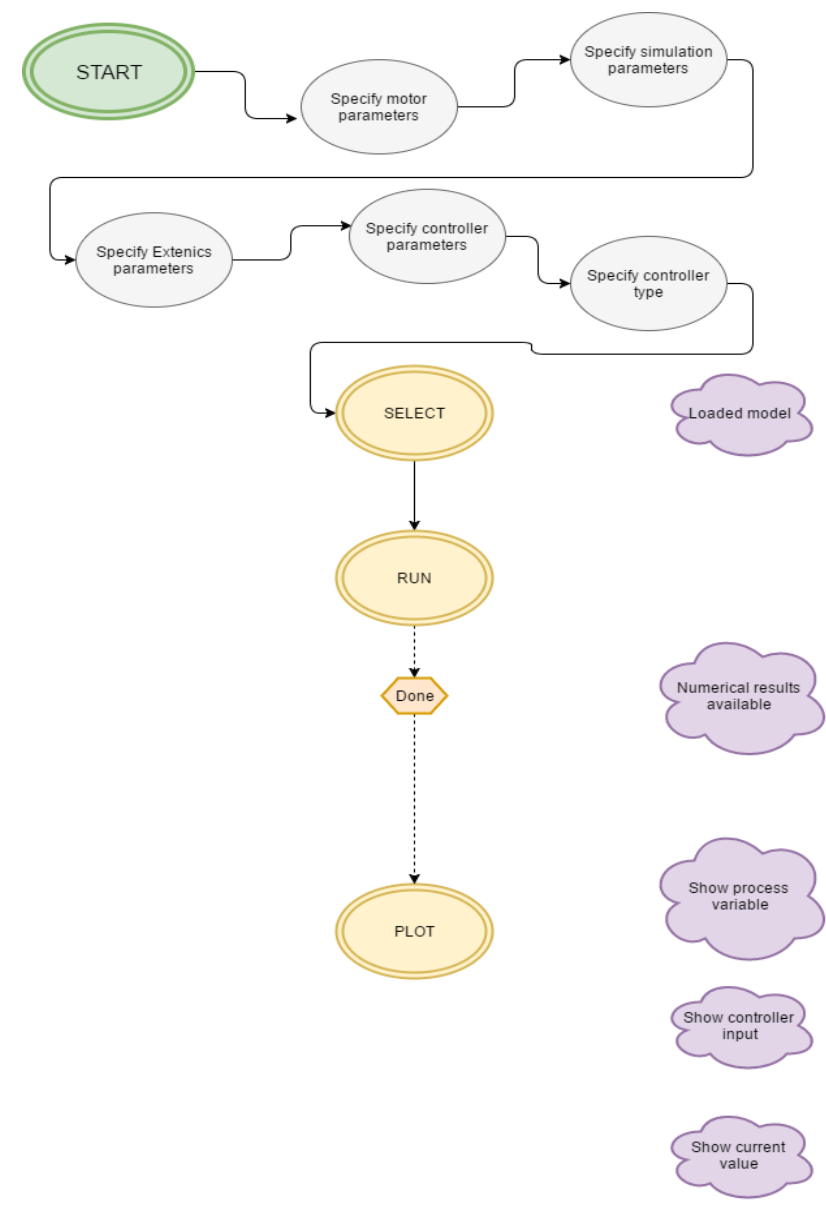

Fig. 3. Application usage diagram.

The application will run the model with the parameters set in the previous steps. As this is an extenics application, i.e. a fuzzy implementation, it is necessary to run the model in Simulink by running the "sim" command (see with the details about the code).

Therefore, the first run of a data set will be slower due to time it takes for Simulink, which runs in background, to initialize, even this application is not visible or available any way to the user.

Without the data obtained by running the simulation, the result pointers cannot be displayed, and trying to visualize the system will end up in an error. Therefore, it is important for the user to be notified after the processing is completed by opening a new window with "Done" message.

At that point, the previously discussed indicators are automatically calculated and displayed and it is possible to visualize the results by pressing "Plot" button.

The first run of a data set will therefore be slower due to the time it takes for Simulink to run in the background, even if the application is not visible or available to the user in any way.

It can be noticed that the application has a linear and intuitive way of working, while at the same time making easier this type of working with this type of modeling, which can be complicated in an unstructured work environment, especially considering the need for parameterization and extended modeling of this type of application.

\section{THE STRUCTURE CODE FOR EXTENICS CONTROL SIMULATION AND VALIDATION (ICEX)}

An extenics application, in the context of the current implementation, is based on fuzzy control, modifying control space according to extenics norms, in both variants of regulators investigated so far. Fig. 4 shows a view of the fuzzy logic part of the current application. The fuzzy implementation associated with this type of regulator has a simple rule base, basically a transformation from the error space into the incompatibility space using the extensions classification. The final effect is more of a smoothing output using fuzzy logic (fuzzy smoothing) being closer to the mathematical norms of extenics, and thus to the classic way of solving a generally incompatible problem in extenics.

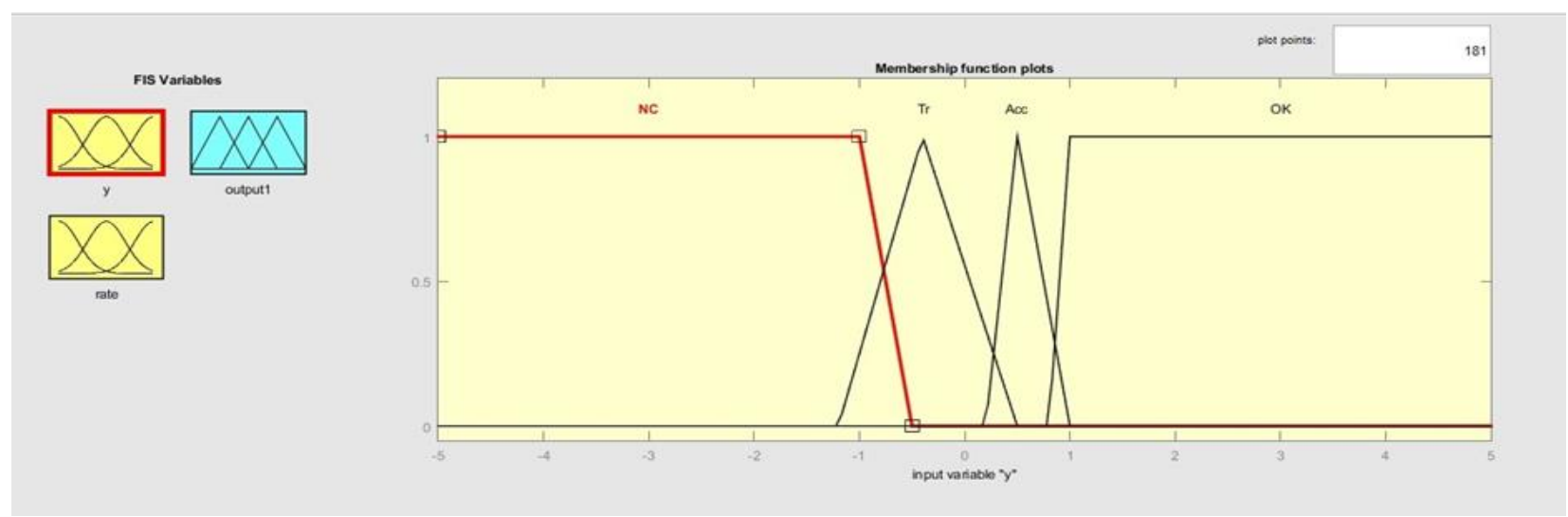

Fig. 4. Structure of fuzzy variable associated with the extended control space.

Enhanced Extended Controller (EEC) adds a new input dimension, the error derivate. The rule base is, of course, more complex and focused on the external action of the controller, and there can be no direct classification in the usual sense of an extenics problem. This controller is a move towards a classic controller, where the main entrance, which remains the error for the process variable, is modelled according to the rules used in extenics.
Fig. 5 shows the Simulink modeling scheme for the enhanced extended controller. The structure loaded in the runtime memory includes the different types of fuzzy structures possible for the two implementations. Although it would have been possible to directly select one of them by the user, the current version of the application automatically runs the best fuzzy inference variant for each option, depending on the user's selection. 


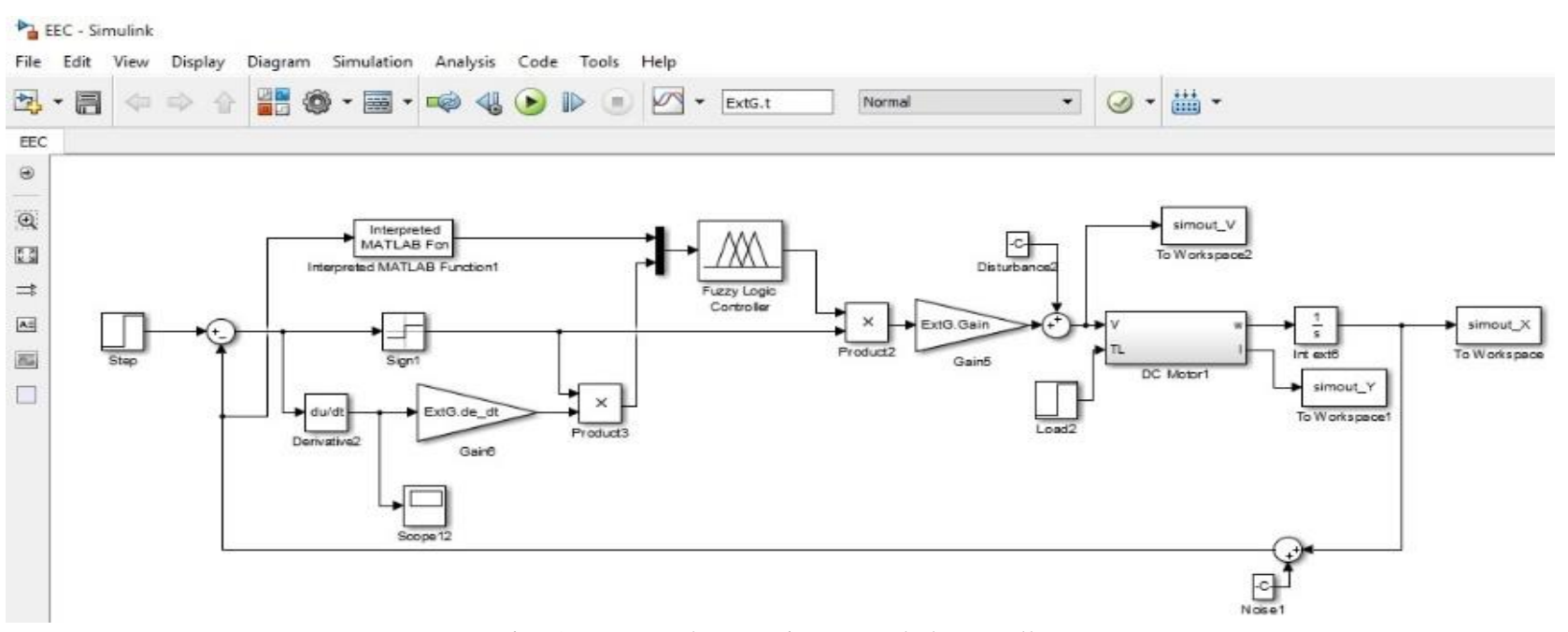

Fig. 5. Control scheme using extended controller.

Because of the operating environment limitations, a complex fuzzy application cannot be run exclusively from the code, but requires a resolving pattern. The data obtained from this model can, however, be loaded directly into a variable that can be called by the application.

Based on this values vector, the calculations required to determine performance indicators can be made, and then to visualize the values of interest in the graphics within the application.

\section{CONCLUSIONS}

The VIPRO interface is a software application that allows the user to access and develop various real time control programs in simulation environment, in virtual environment and/or in real environment. In order to test and validate the developed control method in a real environment in the virtual environment / virtual reality, the user can connect to the VIPRO platform, which is equipped with three action servomotors, three load servomotors six frequency converters acting as high precision positioning controllers, all of these being real time controlled by the PLC system with programmable logic controllers. The user can also connect to NAO robot, which is a component of the VIPRO platform to test the control laws developed by the user.

Modelling a robotic workspace using concepts from Extenics Theory contributes to the development of a new type of innovative control for robot actuators. The advantages of extended control are remarkable through the lack of added complexity in design or implementation. The controller architecture is very straightforward, once the function interpreter is established. While the place and limits of the extended sets need to be specified and may involve some fine tuning, their optimization is not vital, and perfectly fine results can be obtained with simple and intuitive values.

Starting from the theoretical frame and having pillars the basic-element theory, extension set theory and extension logic, the functional and experimental model for robot Extenics simulations applied on VIPRO Platform is leading to the development of an innovative component in artificial intelligence field, the extended control (Extenics), to improve the instantaneous performance of DHFPC control of mobile robots.
For solving contradictory problems is developed an advanced method in the motion control of autonomous robots by applying extension theory on a multi-dimensional space. The obtained results lead to development of a methodology that allows obtaining high level results for robot motion control using extended transformations onto the extension set and an optimization function generated by the extended dependence function in multidimensional space in comparison to the classical method using sequential logic matrices.

\section{ACKNOWLEDGMENT}

This work was developed with the support of MEN-UEFISCDI, PN-II-PT-PCCA-2013-4, VIPRO project No.009/2014-2017, Romanian Academy, “Joint Laboratory of Intelligent Rehabilitation Robot" collaborative research agreement between Romanian Academy by IMSAR, RO and Yanshan University, CN, project KY201501009/2016-2018 and H2020-MSCA-RISE-2016/H2020-MSCA-RISE-2016, SMOOTH project, GA no. 734875 , 2016-2020.

\section{REFERENCES}

[1] C. Y. Yang and W. Cai, Extension Engineering, Science Press, Beijing, 2002.

[2] W. Cai, "Extension set and non-compatible problems," Advances in Applied Mathematics and Mechanics in China, Peking: International Academic Publishers, 1990, pp. 1-21.

[3] V. Vladareanu, O. I. Sandru, P. Schiopu, A. Sandru, and L. Vladareanu, "Extension hybrid force-position control of mechatronics systems," Communications in Cybernetics, Systems Science and Engineering, Beijing: CRC Press, 2013.

[4] L. Vladareanu, G. Tont, I. Ion, V. Vladareanu, and D. Mitroi, "Modeling and hybrid position-force control of walking modular robots," ISI Proceedings, Recent Advances in Applied Mathematics, pp. 510-518, 2010.

[5] O. I. Şandru, L. Vlădăreanu, P. Şchiopu, V. Vlădăreanu, and A. Şandru, "Multidimensional extenics theory," U.P.B. Sci. Bull., Series A, vol. 75, no.1, 2013.

[6] "Florentin Smarandache, Extenics in Higher Dimensions," Institute of Extenics and Innovation Methods, Guangdong University of Technology.

[7] F. Smarandache and L. Vlădăreanu, "Applications of neutrosophic logic to robotics - an introduction," in Proc. the 2011 IEEE International Conference on Granular Computing Kaohsiung, Taiwan, pp. 607-612, Nov. 8-10, 2011.

[8] O. Șandru, L. Vlădăreanu, P. Șchiopu, V. Vlădăreanu, and A. Șandru, "New progress in extenics theory," First International Symposium of Extenics, 2013. 
[9] S. Florentin and V. Victor, "Applications of extenics to 2D-space and 3D-space," in Proc. the 6th Conference on Software, Knowledge, Information Management and Applications, Sept. 9-11, 2012.

[10] F. Smarandache, Generalizations of the distance and dependent function in extenics to 2D, 3D, and n-D. [Online]. Available: http://vixra.org/abs/1206.0014 http://vixra.org/pdf/1206.0014v1.pdf.

[11] V. Vlădăreanu, P. Schiopu, M. C. Deng, and H. N. Yu, "Intelligent extended control of the walking robot motion," in Proc. of the 2014 International Conference on Advanced Mechatronic Systems, pp. 489-495, August 10-12, 2014.

[12] D. Mitroi, "Contributions to new control strategies for autonomous mobile robots using artificial intelligence," Ph.D. dissertation, Institute of Solid Mechanics, Romanian Academy, 2017.

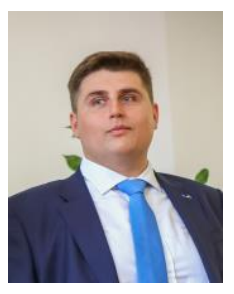

Daniel Mitroi is a Ph.D student in robotics - artificial intelligence in robot control - at the Institute of Solid Mechanics of the Romanian Academy. His scientific work is focused on real time control, mechatronics and robotics, numerical algorithms for mobile robot control. His work is published in 15 scientific papers: ISI, ISI Proceedings, BDI Journals. He was member in 2 FP7 projects and in 2 project financed from National Research, Development and Innovation Plan. He was part of the team rewarded by 3 international prizes and 2 gold medals at International Inventions Saloon in Geneva.

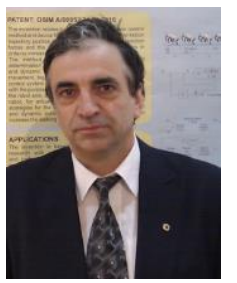

Luige Vlădăreanu received his Ph.D. degree in electronics field, and is the director of Robotics and Mechatronics Department. He is a Ph.D advisor in the field of mechanical engineering at the Romanian Academy and corresponding member of the American-Romanian Academy. He has published over 35 books and book chapters, 11 edited books, over 200 papers in journals, proceedings and conferences in the areas. Director and coordinator of over 15 grants of international and national research - Development programs in the last 5 years, 15 invention patents, developing 17 advanced work methods resulting from applicative research activities and more than 60 research projects. His scientific work is focused on real time control in solid mechanics applied in robotics, mechatronics, complex industrial automations in distributed and decentralized structure.

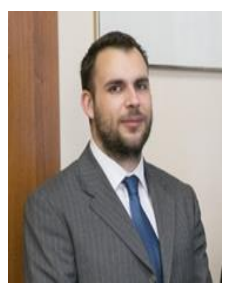

Victor Vlădăreanu is a scientific researcher at the Institute of Solid Mechanics of the Romanian Academy. He has published 5 book chapters, 20 papers in national and international journals, over 30 papers in national and international conferences and is a co-author for international and 6 national patents. Dr. Vlădăreanu has also participated in 3 international and 6 national research grants, and is the legal representative of IMSAR in the "MASIM" project, financed within the national program PN II. He has received his Ph.D from the Faculty of Electronics, with the thesis
"Contributions to the intelligent control of autonomous robots using multi-sensor systems" and his Master's Thesis, from the University of Newcastle, UK, is a comparative study of evolutionary optimization algorithms for the control of autonomous robots. His main research interests are in the field of Robotics and Artificial Intelligence, focusing on fuzzy systems, artificial neural networks, evolutionary algorithms, Extenics, Neutrosophy and analytical statistics.

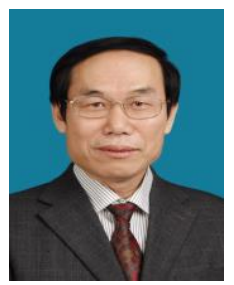

Hongbo Wang received his BS and MS degrees from Institute of Northeast Heavy Machinery, Qiqihar, China in 1982 and 1986. He received his Ph.D degree from Nagasaki University, Nagasaki, Japan in 1997. He studied as a visiting scholar in the Institute of Robotics, ETH Zuerich, Switzerland from 1992 to 1993 . After that, he moved to Nagasaki University as a researcher. From 1998, he worked in Daihen Corporation as a deputy manager, Osaka, Japan. Since 2009, he has been working with Yanshan University, Qinhuangdao, China as a professor. He published about 150 papers in journal and international conference. Since 2012, he presided over The National High Technology Research and Development Program of China, National Key Technology Research and Development Program of China, International Science and Technology Cooperation Program of China. His current research interests are in rehabilitation robot and assisting robot for the disabled and the elderly.

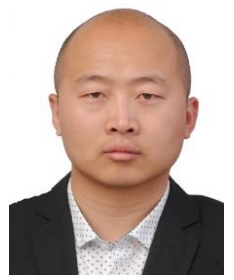

Yongfei Feng, Chinese, was born in 1988. He is currently a joint doctoral-student at Yanshan University, China, and Institute of Solid Mechanics of Romanian Academy, Romania (2016.9-). Once he obtained his master degree at Yanshan University, China, 2015 and the major is mechatronics. His research interests include mechatronics and rehabilitation robot. Dr. Feng is a reviewer of International Journal of Advanced Robotic Systems \&ACTA Automatica Sinica, and so on. He has published more than 8 papers and owned 3 China patents.

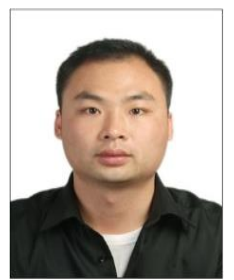

Jianye Niu was born in April 1982. Currently he is a doctoral student of Yanshan University, where he obtained his bachelor degree and master degree of mechatronic engineering. His research interests include robotics, mechanical engineering, neural network and control engineering. E-mail: jyniu@ysu.edu.cn. 